\title{
The loss-of-function PCSK9 p.R46L genetic variant does not alter glucose homeostasis
}

\author{
Amélie Bonnefond ${ }^{1,2,3}$ - Loïc Yengo ${ }^{1,2,3}$ • Cédric Le May ${ }^{4}$. Fréderic Fumeron ${ }^{5,6}$. \\ Michel Marre $^{5,6,7}$ - Beverley Balkau ${ }^{8}$ - Guillaume Charpentier' ${ }^{9}$ Sylvia Franc ${ }^{9}$. \\ Philippe Froguel ${ }^{1,2,3,10} \cdot$ Bertrand Cariou $^{4,11,12}$ - for the DESIR study group
}

Received: 29 March 2015 / Accepted: 13 May 2015 /Published online: 7 June 2015

(C) Springer-Verlag Berlin Heidelberg 2015

\begin{abstract}
Aims/hypothesis Proprotein convertase subtilisin/kexin type 9 (PCSK9) is a critical regulator of cholesterol homeostasis. PCSK9 inhibitors are being actively developed to lower LDL-cholesterol levels. However, there are conflicting data regarding the consequences of Pcsk 9 deficiency on glucose homeostasis in mouse models. Here, we analysed in humans the association between the PCSK9 p.R46L loss-of-function (LOF) variant and (1) glucose homeostasis variables; (2) type 2 diabetes status; and (3) the risk of 9 year incident type 2 diabetes in a prospective study.

Methods PCSK9 p.R46L was genotyped in 4630 French participants from the Data from an Epidemiological Study on the Insulin Resistance Syndrome (DESIR) prospective study and in 1342 French participants with type 2 diabetes. The association between p.R46L and metabolic traits or type 2 diabetes
\end{abstract}

Electronic supplementary material The online version of this article (doi:10.1007/s00125-015-3659-8) contains peer-reviewed but unedited supplementary material, which is available to authorised users.

Philippe Froguel

philippe.froguel@good.ibl.fr

Bertrand Cariou

bertrand.cariou@univ-nantes.fr

1 European Genomic Institute for Diabetes (EGID), FR 3508 Lille, France

2 CNRS UMR 8199, Lille Pasteur Institute, Lille, France

3 Lille University, Lille, France

4 Inserm UMR1087/CNRS UMR6291, l'Institut du Thorax, Nantes, France

5 Inserm U-1138, Centre de Recherche des Cordeliers, Paris, France

6 Sorbonne Paris-Cité, Paris-Diderot University, Paris, France risk was assessed through linear or logistic regression models adjusted for age, sex and BMI. The association between p.R46L and incident type 2 diabetes was assessed using a Cox regression model adjusted for sex, age and BMI at baseline.

Results Significant associations $\left(p<10^{-6}\right)$ were found between p.R46L and lower total cholesterol $(-0.394 \mathrm{mmol} / \mathrm{l})$, LDL-cholesterol $(-0.393 \mathrm{mmol} / \mathrm{l})$ and apolipoprotein B concentrations $(-0.099 \mathrm{~g} / \mathrm{l})$. However, no significant association was observed between p.R46L and markers of glucose homeostasis (including fasting glucose, fasting insulin, $\mathrm{HbA}_{1 \mathrm{c}}$, HOMA-B, HOMA-IR) or type 2 diabetes risk. Furthermore, no significant association between $\mathrm{p} . \mathrm{R} 46 \mathrm{~L}$ variant and risk of incident type 2 diabetes was observed in DESIR.

Conclusions/interpretation The PCSK9 p.R46L LOF variant was not associated with impaired glucose homeostasis in
7 Department of Endocrinology-Diabetology and Nutrition, DHU-FIRE, Bichat Hospital, Assistance Publique-Hôpitaux de Paris, Paris, France

8 Inserm U-1018, CESP, Team 5 (EpReC, Renal and Cardiovascular Epidemiology), UVSQ-UPS, Villejuif, France

9 Department of Diabetes and Endocrinology, Centre Hospitalier Sud-Francilien, Corbeil-Essonne, France

10 Department of Genomics of Common Disease, School of Public Health, Imperial College London, Hammersmith Hospital, London, UK

11 Clinique d'Endocrinologie, l'Institut du Thorax, CHU Nantes, Nantes, France

12 Faculté de Médecine, Université de Nantes, Nantes, France 
humans. These data are reassuring regarding the safety of PCSK9 inhibitors.

Keywords Apolipoprotein B · Cholesterol · LDL . Low-frequency genetic variant $\cdot$ PCSK 9 - Type 2 diabetes

\begin{tabular}{|c|c|}
\hline \multicolumn{2}{|c|}{ Abbreviations } \\
\hline APOA1 & Apolipoprotein A1 \\
\hline APOB & Apoliprotein B \\
\hline DESIR & $\begin{array}{l}\text { Data from an Epidemiological Study on the } \\
\text { Insulin Resistance Syndrome }\end{array}$ \\
\hline DIAGRAM & $\begin{array}{l}\text { Diabetes Genetics Replication and } \\
\text { Meta-analysis }\end{array}$ \\
\hline FPG & Fasting plasma glucose \\
\hline FSI & Fasting serum insulin \\
\hline LDLR & LDL receptor \\
\hline LOF & Loss-of-function \\
\hline MAGIC & $\begin{array}{l}\text { Meta-Analyses of Glucose and Insulin-Related } \\
\text { Traits Consortium }\end{array}$ \\
\hline PCSK9 & Proprotein convertase subtilisin/kexin type 9 \\
\hline
\end{tabular}

\section{Introduction}

Proprotein convertase subtilisin/kexin type 9 (PCSK9) is the ninth member of the proprotein convertase family and is a key regulator of cholesterol homeostasis. PCSK9 is secreted by the liver and acts as an endogenous inhibitor of the LDL receptor (LDLR). PCSK9 loss-of-function (LOF) mutations contribute to low levels of plasma LDL-cholesterol and protection against cardiovascular diseases $[1,2]$. For instance, the PCSK9 LOF low-frequent variant p.R46L (rs11591147; minor allele frequency in the HapMap-CEU (Centre d'Etude du Polymorphisme [Utah residents with northern and western European ancestry] population: $2.8 \%$ ) has been shown to increase the number of cell surface LDLRs in hepatocytes in vitro [3], leading to a mean reduction in LDL-cholesterol of $9 \%$ to $16 \%$ in p.R $46 \mathrm{~L}$ carriers. Recently, several Phase 3 trials have been conducted with human monoclonal antibodies directed against PCSK9 in patients with cardiovascular diseases, or familial or primary hypercholesterolaemia. PCSK9 inhibition has led to a drastic and consistent reduction in baseline LDL-cholesterol levels of up to $\sim 60 \%$, independent of background lipid-lowering therapy [4].

As well as being expressed mainly in the liver, PCSK9 is also expressed in mouse and human pancreatic islets and is able to downregulate the LDLR in human isolated islets [5]. Since it has been suspected that LDLR mediated lipotoxicity can alter beta cell function [6], a potential safety issue with the use of PCSK9 inhibitors is the increased risk of type 2 diabetes. However, results obtained from Pcsk9-deficient mice are discordant. One study found no deleterious effect of $P c s k 9$ deficiency on glucose homeostasis [5], while another demonstrated that Pcsk9-deficient mice are glucose intolerant with reduced insulin secretion [7].

The aim of the present study was to assess the associations between the PCSK9 p.R46L variant and (1) changes in several glucose and lipid homeostasis variables in non-diabetic French participants; (2) type 2 diabetes in a French case-control study; (3) the incidence of type 2 diabetes over a 9 year follow-up period.

\section{Methods}

Study populations Genotyping of $p . R 46 \mathrm{~L}$ was performed in two European-French populations: the Data from an Epidemiological Study on the Insulin Resistance Syndrome (DESIR) and Corbeil studies.

The DESIR cohort is a 9 year longitudinal study in a French general population. We analysed $4630 \mathrm{DESIR}$ participants successfully genotyped for p.R46L, of whom 4319 were nondiabetic during the follow-up period, 2207 had normal glucose homeostasis (and were used as controls in the case-control study), 127 presented with type 2 diabetes at baseline (and were used as cases in the case-control study) and 184 developed type 2 diabetes during the 9 year follow-up.

The Corbeil study included 1342 unrelated French type 2 diabetes patients successfully genotyped for p.R46L who were recruited by the Endocrinology-Diabetology Department of the Corbeil-Essonnes Hospital (Corbeil-Essonnes, France).

Glycaemic status was defined according to 1997 ADA criteria; normal glucose homeostasis was defined as a fasting plasma glucose (FPG) level $<6.1 \mathrm{mmol} / 1$ without glucoselowering treatment, and type 2 diabetes was defined as FPG $\geq 7.0 \mathrm{mmol} / \mathrm{l}$ or treatment with glucose-lowering agents. Nondiabetic participants had $\mathrm{FPG}<7 \mathrm{mmol} / \mathrm{l}$ and were not treated with glucose-lowering agents.

The study protocol was approved by the local ethics committees and all participants provided written informed consent.

Genotyping of p.R46L variant Genotyping of the p.R46L variant (rs11591147) was performed using Metabochip DNA arrays (Illumina, San Diego, CA, USA), as previously described [8]. The individual call rate for the DESIR and Corbeil participants was $>98 \%$ for the p.R46L variant and no departures from the Hardy-Weinberg equilibrium were observed in either study $(p>0.05)$. Cluster plots are shown in electronic supplementary material (ESM) Fig. 1.

Index calculation HOMA-B and HOMA-IR were calculated as previously described [9]. 
Statistical analyses The associations between p.R46L and quantitative traits was assessed in 4319 non-diabetic participants from DESIR through linear regression models adjusted for age, sex and BMI (except for the analysis of BMI, which was adjusted for age and sex only).

The case-control analysis included type 2 diabetes participants from DESIR (at baseline) and from Corbeil, and normal glucose controls from DESIR (during the follow-up period). The association between p.R46L and type 2 diabetes was assessed using a logistic regression model adjusted for age, sex and BMI.

The association between p.R46L and incident type 2 diabetes was assessed in DESIR using a Cox regression model adjusted for sex, age and BMI at baseline, and the timescale used was age.

All the analyses were performed under an additive model.

Given the size of our studies, we would have been able to detect a significant OR of 1.55 (type 2 diabetes case-control study) and a significant HR of 4.40 (incident type 2 diabetes study), with a power of $80 \%$ (ESM Fig. 2).

Except for statistical power analyses, we performed statistical analyses using SPSS (version 14.0), IBM, Armonk, NY, USA.

\section{Results}

In the 4,319 non-diabetic participants from the DESIR study, we assessed the association of PCSK9 p.R46L with BMI, glucose homeostasis related traits (including FPG, FSI, HOMA-B, HOMA-IR and, $\mathrm{HbA}_{1 \mathrm{c}}$ ), lipid homeostasis variables (including total cholesterol, HDL-cholesterol, LDL-cholesterol, triacylglycerol, apolipoprotein B [APOB] and apolipoprotein A1 [APOA1]) and liver enzyme levels (alanine aminotransferase and aspartate aminotransferase [Table 1]). When we adjusted our models for age, sex and BMI, we confirmed a marked association between the p.R $46 \mathrm{~L}$ variant and decreased total cholesterol levels (effect size $[95 \% \mathrm{CI}]-0.394[-0.537$, $-0.251] \mathrm{mmol} / \mathrm{l} ; p=7.05 \times 10^{-8}$ ), decreased LDL-cholesterol levels (effect size $-0.393[-0.525,-0.261] \mathrm{mmol} / 1 ; p=$ $5.18 \times 10^{-9}$ ) and decreased APOB levels (effect size -0.099 $[-0.136,-0.061] \mathrm{g} / 1 ; p=2.42 \times 10^{-7}$ [Table 1]). These association results were similar when the models were adjusted for age, sex, BMI and lipid-lowering medication (data not shown). However, we did not find any significant association between p.R46L and variations in other traits (Table 1). According to the Meta-analyses of Glucose and Insulin-Related Traits Consortium (MAGIC), in $>133,000$ non-diabetic participants, p.R46L was nominally (but not significantly) associated with increase fasting glucose levels (effect size 0.028 $[-0.006,0.050] \mathrm{mmol} / \mathrm{l} ; p=0.013)$, and it was not associated with fasting insulin levels (effect size $0.016[-0.008,0.040]$ $\mathrm{pmol} / 1 ; p=0.16$ ) [10].
Of note, in the 4,319 non-diabetic participants from DESIR, there was no significant difference regarding statin use between p.R46L carriers $(n=4$ of 171 [2.3\%] participants informed for statin use) and non-carriers ( $n=108$ out of 4,082 [2.6\%] participants informed for statin use; $p=0.81$ ).

In the type 2 diabetes case-control study, we did not find any significant association between p.R46L and type 2 diabetes $(p=0.261)$ or incident type 2 diabetes over the 9 years of follow-up ( $p=0.065$ [Table 2]). In addition, there was no significant association between p.R46L and occurrence of impaired fasting glucose (FPG $\geq 6.1 \mathrm{mmol} / \mathrm{l} ; n=508$ incident cases) over the 9 years of follow-up (HR [95\% CI] 0.91 $[0.58,1.42] ; p=0.66$; data not shown). According to the Diabetes Genetics Replication and Meta-analysis (DIAGRAM) consortium, p.R46L was not associated with type 2 diabetes risk in a total of 42,590 European participants (OR [95\% CI] 1.09 [0.98, 1.22]; $p=0.12$ ) [11].

\section{Discussion}

The major finding of this study is that the PCSK9 p.R46L LOF variant was not associated with impaired glucose homeostasis in humans. Notably, we showed that p.R46L carriers did not have an increased incidence of type 2 diabetes during a 9 year follow-up or type 2 diabetes risk in a case-control study.

Although positive correlations were observed between plasma PCSK9 and both FPG and HOMA-IR in the Dallas Heart Study [12], we did not find any associations between p.R46L and either FPG, $\mathrm{HbA}_{1 \mathrm{c}}$, or markers of insulin resistance (HOMA-IR) or insulin secretion (HOMA-B). However, a non-clinically relevant tendency for a positive association between p.R46L and FPG was observed in MAGIC. As expected [1,2], p.R46L remained associated with markers of LDL metabolism with reduced total cholesterol, LDLcholesterol and APOB concentrations in people carrying the p.R46L allele. The use of statins is associated with an increased risk of type 2 diabetes and could be a potential confounding factor [13]. However, adding lipid-lowering medication status into the model did not modify the results.

The present results are in line with our previous results obtained in PCSK9-deficient mice, in which there was no alteration of glucose homeostasis, as assessed by both glucose and insulin tolerance tests [5]. While PCSK9 deficiency is associated with an increased expression of LDLR in pancreatic islets, there is no increase in intracellular cholesterol content in PCSK9-deficient islets, even under lipotoxic conditions when incubating isolated islets with high concentrations of LDL [5]. This absence of intracellular cholesterol accumulation might be due to reduced circulating LDL levels in PCSK9-deficient mice and/or to an increase in cholesterol efflux from beta cell. 
Table 1 Associations between the PCSK9 p.R46L variant and glucose homeostasis related traits, lipid homeostasis variables and liver enzyme levels in 4,319 non-diabetic participants from DESIR

\begin{tabular}{|c|c|c|c|c|c|}
\hline \multirow[t]{2}{*}{ Clinical data } & \multirow[t]{2}{*}{$n$} & \multicolumn{2}{|c|}{ Mean/median data level by genotype ${ }^{\mathrm{a}}$} & \multirow[t]{2}{*}{ Effect size $(95 \% \mathrm{CI})$} & \multirow[t]{2}{*}{$p$ value } \\
\hline & & GG & GT & & \\
\hline $\begin{array}{l}\text { Sex } \\
(M / F)\end{array}$ & $2,073 / 2,246$ & $1,980 / 2,166$ & $93 / 80$ & - & - \\
\hline $\begin{array}{l}\text { Age } \\
\text { (years) }\end{array}$ & 4,319 & $46.7 \pm 10.0$ & $47.9 \pm 10.1$ & - & - \\
\hline $\begin{array}{l}\mathrm{BMI} \\
\left(\mathrm{kg} / \mathrm{m}^{2}\right)\end{array}$ & 4,253 & $24.5 \pm 3.7$ & $24.7 \pm 3.7$ & $\begin{array}{l}0.046 \\
(-0.49,0.58)\end{array}$ & 0.865 \\
\hline $\begin{array}{l}\text { FPG } \\
(\mathrm{mmol} / \mathrm{l})\end{array}$ & 4,313 & $5.24 \pm 0.55$ & $5.29 \pm 0.54$ & $\begin{array}{l}0.013 \\
(-0.026,0.052)\end{array}$ & 0.736 \\
\hline $\begin{array}{l}\text { FSI } \\
(\mathrm{pmol} / \mathrm{l})\end{array}$ & 4,316 & $\begin{array}{l}38.9 \\
(28.5-54.7)\end{array}$ & $\begin{array}{l}39.6 \\
(28.0-59.6)\end{array}$ & $\begin{array}{l}0.006 \\
(-0.062,0.074)\end{array}$ & 0.864 \\
\hline HOMA-B & 4,310 & $\begin{array}{l}67.4 \\
(48.9-95.1)\end{array}$ & $\begin{array}{l}68.2 \\
(46.3-95.9)\end{array}$ & $\begin{array}{l}-0.003 \\
(-0.040,0.033)\end{array}$ & 0.927 \\
\hline HOMA-IR & 4,310 & $\begin{array}{l}1.30 \\
(0.92-1.90)\end{array}$ & $\begin{array}{l}1.30 \\
(0.92-2.04)\end{array}$ & $\begin{array}{l}0.008 \\
(-0.030,0.046)\end{array}$ & 0.833 \\
\hline $\begin{array}{l}\mathrm{HbA}_{1 \mathrm{c}} \\
(\%)\end{array}$ & 4,314 & $5.25 \pm 0.41$ & $5.28 \pm 0.39$ & $\begin{array}{l}0.008 \\
(-0.050,0.066)\end{array}$ & 0.788 \\
\hline $\begin{array}{l}\mathrm{HbA}_{1 \mathrm{c}} \\
(\mathrm{mmol} / \mathrm{mol})\end{array}$ & 4,314 & $33.9 \pm 4.5$ & $34.2 \pm 4.3$ & $\begin{array}{l}0.086 \\
(-0.543,0.716)\end{array}$ & 0.788 \\
\hline $\begin{array}{l}\mathrm{TC} \\
(\mathrm{mmol} / \mathrm{l})\end{array}$ & 4,319 & $5.74 \pm 1.00$ & $5.39 \pm 0.95$ & $\begin{array}{l}-0.394 \\
(-0.537,-0.251)\end{array}$ & $7.05 \times 10^{-8}$ \\
\hline HDL-C (mmol/l) & 4,278 & $1.65 \pm 0.43$ & $1.63 \pm 0.42$ & $\begin{array}{l}-0.004 \\
(-0.064,0.055)\end{array}$ & 0.890 \\
\hline LDL-C (mmol/l) & 4,278 & $3.58 \pm 0.92$ & $3.24 \pm 0.87$ & $\begin{array}{l}-0.393 \\
(-0.525,-0.261)\end{array}$ & $5.18 \times 10^{-9}$ \\
\hline $\begin{array}{l}\mathrm{TG} \\
(\mathrm{mmol} / \mathrm{l})\end{array}$ & 4,319 & $\begin{array}{l}0.93 \\
(0.67-1.35)\end{array}$ & $\begin{array}{l}0.98 \\
(0.67-1.49)\end{array}$ & $\begin{array}{l}0.012 \\
(-0.061,0.085)\end{array}$ & 0.744 \\
\hline $\begin{array}{l}\text { APOB } \\
(\mathrm{g} / \mathrm{l})\end{array}$ & 4,318 & $1.09 \pm 0.27$ & $1.00 \pm 0.26$ & $\begin{array}{l}-0.099 \\
(-0.136,-0.061)\end{array}$ & $2.42 \times 10^{-7}$ \\
\hline $\begin{array}{l}\text { APOA1 } \\
(\mathrm{g} / \mathrm{l})\end{array}$ & 4,318 & $1.65 \pm 0.26$ & $1.64 \pm 0.24$ & $\begin{array}{l}-0.001 \\
(-0.039,0.036)\end{array}$ & 0.942 \\
\hline $\begin{array}{l}\text { ALAT } \\
(\mathrm{U} / \mathrm{I})\end{array}$ & 4,316 & $\begin{array}{l}21.3 \\
(15.8-29.5)\end{array}$ & $\begin{array}{l}21.8 \\
(15.8-31.5)\end{array}$ & $\begin{array}{l}0.024 \\
(-0.041,0.089)\end{array}$ & 0.474 \\
\hline $\begin{array}{l}\mathrm{AST} \\
(\mathrm{U} / \mathrm{I})\end{array}$ & 4,316 & $\begin{array}{l}18.6 \\
(15.0-23.2)\end{array}$ & $\begin{array}{l}18.6 \\
(15.8-22.9)\end{array}$ & $\begin{array}{l}-0.011 \\
(-0.063,0.041)\end{array}$ & 0.689 \\
\hline
\end{tabular}

${ }^{\mathrm{a}}$ Data are means \pm SD or, for logarithmically transformed variables (including FSI, HOMA-B, HOMA-IR, TG, ALAT and AST), data are median (25th75th percentile)

ALAT, alanine aminotransferase; AST, aspartate aminotransferase; HDL-C, HDL-cholesterol; LDL-C, LDL-cholesterol; TC, total cholesterol; TG, triacylglycerol

Table 2 Association between the PCSK9 p.R46L variant and type 2 diabetes risk and incidence

\begin{tabular}{|c|c|c|c|c|c|c|c|}
\hline & $n$ & T-allele frequency & GG & GT & TT & OR or $\operatorname{HR}(95 \% \mathrm{CI})$ & $p$ value \\
\hline \multicolumn{8}{|l|}{ Type 2 diabetes case-control analysis } \\
\hline $\begin{array}{l}\text { Normal glucose controls from DESIR } \\
\text { Type } 2 \text { diabetes cases from Corbeil study and DESIR }\end{array}$ & $\begin{array}{l}2,207 \\
1,469\end{array}$ & $\begin{array}{l}2.15 \% \\
1.97 \%\end{array}$ & $\begin{array}{l}2,112 \\
1,412\end{array}$ & $\begin{array}{l}95 \\
56\end{array}$ & $\begin{array}{l}0 \\
1\end{array}$ & $\begin{array}{l}0.81 \\
(0.56-1.17)^{\mathrm{a}}\end{array}$ & 0.261 \\
\hline \multicolumn{8}{|l|}{ Type 2 diabetes incidence analysis } \\
\hline $\begin{array}{l}\text { Non-diabetic participants from DESIR } \\
\text { Incident type } 2 \text { diabetes cases from DESIR }\end{array}$ & $\begin{array}{l}4,280 \\
184\end{array}$ & $\begin{array}{l}2.09 \% \\
0.83 \%\end{array}$ & $\begin{array}{l}4,108 \\
181\end{array}$ & $\begin{array}{l}172 \\
3\end{array}$ & $\begin{array}{l}0 \\
0\end{array}$ & $\begin{array}{l}0.34 \\
(0.11-1.07)^{b}\end{array}$ & 0.065 \\
\hline
\end{tabular}

Data are $n$ unless otherwise indicated

${ }^{\mathrm{a}}$ OR from a logistic regression model adjusted for age, sex and BMI

${ }^{\mathrm{b}} \mathrm{HR}$ from a Cox regression model adjusted for age, sex and BMI at baseline 
Our study has several limitations that should be highlighted. First, we only focussed on the effect of the most frequent PCSK9 LOF variant, i.e. p.R46L, and we cannot exclude the possibility that other rare PCSK9 LOF mutations with stronger functional effects may alter glucose homeostasis. Second, the intensity of PCSK9 inhibition observed in heterozygous carriers of the p.R46L variant is inferior to those obtained with PCSK9 monoclonal antibodies. Indeed, the decrease of LDL-cholesterol is $10 \%$ in p.R46L carriers compared with $\sim 60 \%$ with alirocumab or evolocumab in Phase 2 trials [4]. However, such a small decrease in LDLcholesterol was consistently associated with a highly significant reduction of cardiovascular events in individuals carrying the p.R46L allele $[1,2]$, certainly because it was maintained throughout their lives.

In conclusion, a genetic PCSK9 deficiency is not associated with an increased risk of type 2 diabetes. These data are reassuring regarding the safety of PCSK9 inhibitors. Precise glucose phenotyping of individuals treated with PCSK9 monoclonal antibodies in ongoing Phase 3 trials will give some additional insights into the relationship between PCSK9 and glucose homeostasis.

Acknowledgements We are sincerely indebted to all participants in the present study. We thank M. Deweirder and F. Allegaert (EGID, Lille) for their invaluable management of DNA samples. Data from the DIAG RAM consortium on type 2 diabetes have been downloaded from www.diagram-consortium.org. Data from MAGIC on fasting glucose and fasting insulin have been downloaded from www. magicinvestigators.org. The DESIR study group. Inserm U1018: B. Balkau, P. Ducimetière and E. Eschwège; Inserm U367: F. AlhencGelas; CHU D'Angers: Y Gallois and A. Girault; Bichat Hospital: F. Fumeron, M. Marre and R. Roussel; CHU de Rennes: F. Bonnet; CNRS UMR8090, Lille: S. Cauchi and P. Froguel; Centres d'Examens de Santé: Alençon, Angers, Blois, Caen, Chateauroux, Chartres, Cholet, Le Mans, Orléans, Tours; Institute de Recherche Médecine Générale: J. Cogneau; General practitioners of the region; Institute inter-Regional pour la Santé: C. Born, E. Caces, M. Cailleau, O. Lantieri, J. G. Moreau, F. Rakotozafy, J. Tichet.

Funding This work was supported by the Fondation Leducq (Transatlantic Network of Excellence Programme: 'The function and regulation of PCSK9: a novel modulator of LDLR activity', to BC), Fundazione Cariplo (to BC), Contrat de Projets Etat-Région Nord-Pas-De Calais (CPER Axe Cardio-Diabète, to PF), Délégation Régionale à la Recherche et à la Technologie de la Région Nord-Pas-De-Calais (to PF), and the European Union (Fonds Européen de Développement Régional, to PF). $\mathrm{AB}$ was supported by Inserm. The DESIR study was funded by Inserm contracts with Caisse Nationale de l'Assurance Maladie des Travailleurs Salariés (CNAMTS), Lilly, Novartis Pharma, and Sanofi-Aventis, Inserm (Réseaux en Santé Publique, Interactions Entre les Déterminants de la Santé, Cohortes Santé TGIR 2008), the Association Diabète Risque Vasculaire, the Fédération Française de Cardiologie, La Fondation de France, Association de Langue Française pour l'Etude du Diabète et des Maladies Métaboliques (ALFEDIAM)/Société Francophone de Diabétologie, L'Office National Interprofessionnel des Vins (ONIVINS),
Ardix Medical, Bayer Diagnostics, Becton Dickinson, Cardionics, Merck Santé, Novo Nordisk, Pierre Fabre, Roche, and Topcon.

Contribution statement $\mathrm{AB}$ designed the study, performed the statistical analyses and wrote the manuscript. LY contributed to statistical analyses and contributed to the discussion. CLM, FF, MM, BB, GC and SF contributed to acquisition of data and reviewed/edited the manuscript. PF contributed to acquisition of data, reviewed/edited the manuscript and is one of the guarantors of this work. BC designed the study, wrote the manuscript and is one the guarantors of this work. All the authors approved the final version of the submitted draft.

Duality of interest $\mathrm{BC}$ has received advisory board fees from Amgen and Sanofi/Regeneron Pharmaceuticals. The other authors declare that there is no duality of interest associated with this manuscript.

\section{References}

1. Cohen JC, Boerwinkle E, Mosley TH, Hobbs HH (2006) Sequence variations in PCSK9, low LDL, and protection against coronary heart disease. N Engl J Med 354:1264-1272

2. Benn M, Nordestgaard BG, Grande P et al (2010) PCSK9 R46L, low-density lipoprotein cholesterol levels, and risk of ischemic heart disease: 3 independent studies and meta-analyses. J Am Coll Cardiol 55:2833-2842

3. Cameron J, Holla ØL, Ranheim Tet al (2006) Effect of mutations in the PCSK9 gene on the cell surface LDL receptors. Hum Mol Genet $15: 1551-1558$

4. Farnier M (2013) PCSK9 inhibitors. Curr Opin Lipidol 24:251-258

5. Langhi C, Le May C, Gmyr V et al (2009) PCSK9 is expressed in pancreatic delta-cells and does not alter insulin secretion. Biochem Biophys Res Commun 390:1288-1293

6. von Eckardstein A, Sibler RA (2011) Possible contributions of lipoproteins and cholesterol to the pathogenesis of diabetes mellitus type 2. Curr Opin Lipidol 22:26-32

7. Mbikay M, Sirois F, Mayne J et al (2010) PCSK9-deficient mice exhibit impaired glucose tolerance and pancreatic islet abnormalities. FEBS Lett 584:701-706

8. Vaxillaire M, Yengo L, Lobbens S et al (2014) Type 2 diabetesrelated genetic risk scores associated with variations in fasting plasma glucose and development of impaired glucose homeostasis in the prospective DESIR study. Diabetologia 57:1601-1610

9. Matthews DR, Hosker JP, Rudenski AS et al (1985) Homeostasis model assessment: insulin resistance and beta-cell function from fasting plasma glucose and insulin concentrations in man. Diabetologia 28:412-419

10. Scott RA, Lagou V, Welch RP et al (2012) Large-scale association analyses identify new loci influencing glycemic traits and provide insight into the underlying biological pathways. Nat Genet 44:991-1005

11. Morris AP, Voight BF, Teslovich TM et al (2012) Large-scale association analysis provides insights into the genetic architecture and pathophysiology of type 2 diabetes. Nat Genet 44:981-990

12. Lakoski SG, Lagace TA, Cohen JC et al (2009) Genetic and metabolic determinants of plasma PCSK9 levels. J Clin Endocrinol Metab 94:2537-2543

13. Sattar N, Preiss D, Murray HM et al (2010) Statins and risk of incident diabetes: a collaborative meta-analysis of randomised statin trials. Lancet 375:735-742 